\title{
SECOND-ORDER TIME DISCRETIZATION WITH FINITE-ELEMENT METHOD FOR PARTIAL INTEGRO-DIFFERENTIAL EQUATIONS WITH A WEAKLY SINGULAR KERNEL
}

\author{
CHANG HO KIM ${ }^{1}$ and $\mathrm{U}$ JIN CHOI ${ }^{2}$
}

(Received 8 July 1996; revised 23 June 1997)

\begin{abstract}
We propose the second-order time discretization scheme with the finite-element approximation for the partial integro-differential equations with a weakly singular kernel. The space discretization is based on the finite element method and the time discretization is based on the Crank-Nicolson scheme with a graded mesh. We show the stability of the scheme and obtain the second-order convergence result for the fully discretized scheme.
\end{abstract}

\section{Introduction}

We consider the time discretization method for the following partial integro-differential equation with a weakly singular kernel:

$$
\begin{aligned}
u_{t}-\mathscr{A} u(t) & =\int_{0}^{t} K(t-s) \mathscr{B} u(s) d s+f(x, t), \quad x \in \Omega, \quad \text { for } t>0, \\
u & =0, \quad \text { on } \partial \Omega, t>0 \\
u(x, 0) & =u_{0}(x), \quad \text { in } \Omega,
\end{aligned}
$$

where $\mathscr{A}$ is a linear positive self-adjoint elliptic operator, $\mathscr{B}$ is a general partial differential operator of second order with smooth and time-independent coefficients and $K$ is a weakly singular kernel satisfying

$$
\left|K^{i}(t)\right| \leq C_{K} t^{-i-\alpha} \quad \text { with } 0 \leq \alpha<1, \quad \text { for } \quad t>0, \quad i=0,1 .
$$

\footnotetext{
'Department of Applied Mathematics of Kon-Kuk University, Danwol 322, Chungju 380-701, Korea. ${ }^{2}$ Department of Mathematics of KAIST, Kusong-Dong 373-1, Yousong-Gu, Taejon 305-701, Korea. (C) Australian Mathematical Society 1999, Serial-fee code 0334-2700/99
} 
Furthermore, throughout this paper, $\Omega$ is a sufficiently smooth domain in $\mathbb{R}^{d}, d \geq 1$ and we assume that $f$ is sufficiently smooth. Problems of this nature arise in several areas, such as the theory of linear viscoelasticity and heat conduction in material with memory; see, for example, [8].

The numerical method considered in this paper is obtained by discretizing in space by the finite-element method, followed by a finite difference and quadrature scheme for the time discretization. For the numerical solutions we assume that we are given a family $\left\{S_{h}\right\}$ of finite-dimensional subspaces of $H_{0}^{1}=H_{0}^{1}(\Omega)$ such that

$$
\inf _{\chi \in S_{h}}\left\{\|v-\chi\|+h\|v-\chi\|_{1}\right\} \leq C h^{2}\|v\|_{2}, \quad \forall v \in H^{2} \cap H_{0}^{1},
$$

where $\|\cdot\|$ is the norm in $L_{2}=L_{2}(\Omega)$ and $\|\cdot\|_{s}$ is that in $H^{s}=H^{s}(\Omega)$.

As a starting point for the discretization of (1.1), we define the semi-discrete solution of (1.1) as the function $u_{h}:(0, T] \rightarrow S_{h}$ such that

$$
\begin{aligned}
\left(u_{h, t}, \chi\right)+A\left(u_{h}, \chi\right) & =\int_{0}^{t} K(t-s) B\left(u_{h}(s), \chi\right) d s+(f(t), \chi), \forall \chi \in S_{h}, t>0 \\
u_{h}(0) & =u_{0 h},
\end{aligned}
$$

where $(\cdot, \cdot)$ is the inner product in $L_{2}, A(\cdot, \cdot)$ and $B(\cdot, \cdot)$ are the bilinear forms on $H_{0}^{1}$ associated with the differential operators $\mathscr{A}$ and $\mathscr{B}$ and where $u_{0 h}$ is an appropriate approximation in $S_{h}$ of initial data in (1.1). In [2], we can find that for each $T>0$, the error estimate of (1.3) is

$$
\left\|u_{h}(t)-u(t)\right\| \leq C_{T} h^{2}\left\{\left\|u_{0}\right\|_{2}+\int_{0}^{t}\left\|u_{t}\right\| d s\right\} \quad \text { for } \quad t \leq T .
$$

The time-discretization of (1.1) is very interesting because of the nature of "memory effect". The time discretization methods are derived essentially by replacing the derivatives in (1.3) by a difference quotient and using a quadrature rule for the integral terms. The difficulties involved in such time discretization are that all the values of $u(t)$ have to be retained, causing great demands for data storage. To overcome this difficulty, higher-order quadrature formulae or quadrature based on the use of sparser sets of time levels were proposed in literature such as [6,7] and [9] for partial integrodifferential equations with smooth kernels. In the case of weakly singular kernels, the regularity of the solution with respect to time is limited, which makes higher-order quadrature formulae useless, as well as quadratures based on the use of a sparser set of time levels. In fact, for sufficiently smooth data $u_{0}$ and $f$, there exists a unique solution of (1.1) satisfying the following regularities (see [2]):

$$
\begin{gathered}
u \in C\left([0, T] ; H^{2} \cap H_{0}^{1}\right), \quad u_{t} \in C\left([0, T] ; L_{2}\right) \cap L_{1}\left(0, T ; H^{2} \cap H_{0}^{1}\right), \\
u_{t} \in L_{1}\left(0, T ; L_{2}\right) .
\end{gathered}
$$


Formally the solution of (1.1) satisfies

$$
u_{t}=\mathscr{A} u_{t}+K(t) \mathscr{B} u(0)+\int_{0}^{t} K(t-s) \mathscr{B} u_{s} d s+f_{t}
$$

or

$$
\left|u_{t \prime}\right| \leq C_{K} t^{-\alpha}|\mathscr{B} u(0)|+\text { more regular terms with respect to } t
$$

In advance, we assume that the solution of (1.1) satisfies

$$
\left\|u_{1 r}\right\| \leq R_{0} t^{-\alpha} \text { and }\left\|u_{11}\right\|_{2} \leq R_{0} t^{-\alpha} \text { for some } R_{0}>0 \text {. }
$$

Furthermore, it is an easy consequence of (1.4) that

$$
\left\|u_{t \prime}\right\| \leq C t^{-1-\alpha} \text { for some } C>0 .
$$

In this paper, we consider the graded mesh for the discretization of (1.3) (see also [1] and [3]). Given $M \in \mathbb{N}$, let $\Pi_{M}:=\left\{t_{0}, \ldots, t_{M}\right\},\left(0=t_{0}<t_{1}<\cdots<t_{M}=T\right)$, denote a partition of the interval $[0, T]$. With a given partition $\Pi_{M}$ of $[0, T]$ we associate the quantities

$$
\bar{k}:=\max _{n \leq M} k_{n},
$$

where $k_{n}:=t_{n}-t_{n-1}(n=1, \ldots, M)$. If the mesh points $\left\{t_{n}\right\}_{n=0}^{M}$ are given by

$$
t_{n}:=\left(\frac{n}{M}\right)^{r} T \quad(n=0, \ldots, M),
$$

then $\Pi_{M}$ is called a graded mesh; in the present context, the so-called grading exponent $r \in \mathbb{R}$ will always satisfy $r \geq 1$. Let $U^{n} \in S_{h}$ be the approximation of the exact solution of (1.3) at time $t_{n}$. The time discretization considered here is based on the backward-difference quotient $\bar{\partial}_{t} U_{n}=\left(U^{n}-U^{n-1}\right) / k_{n}$. The integral term then has to be evaluated by numerical quadrature from the values of the $U^{j} \mathrm{~s}$, but since the integrand is singular, we use product integration. We approximate $\phi$ in $J_{n}(\phi)=$ $\int_{0}^{t_{n}} K\left(t_{n}-s\right) \phi(s) d s$ by piecewise functions

$$
\tilde{\phi}(s)= \begin{cases}\phi^{0} & s \in\left(0, t_{1}\right], \\ \phi^{j+1 / 2} & s \in\left(t_{j}, t_{j+1}\right], \quad 1 \leq j \leq n-2, \\ \frac{t_{n-1 / 2}-s}{k_{n-1 / 2}} \phi^{n-3 / 2}+\frac{s-t_{n-3 / 2}}{k_{n-1 / 2}} \phi^{n-1 / 2} & s \in\left(t_{n-1}, t_{n}\right], \quad n \geq 2,\end{cases}
$$


where we denote that $t_{j-1 / 2}:=\left(t_{j}+t_{j-1}\right) / 2, \phi^{j-1 / 2}:=\left(\phi^{j}+\phi^{j-1}\right) / 2$ and $k_{j-1 / 2}:=$ $\left(k_{j}+k_{j-1}\right) / 2$. Thus we write the quadrature for $J_{n}(\phi)$ as

$$
\begin{aligned}
q^{n}(\phi) & =\sum_{j=0}^{n-1} \int_{t_{j}}^{t_{j+1}} K\left(t_{n}-s\right) \tilde{\phi}(s) d s \\
& =\tau_{n 0} \phi^{0}+\sum_{j=1}^{n-2} \tau_{n j} \phi^{j+1 / 2}+\tau_{n n-1} \phi^{n-3 / 2}+\tau_{n n} \phi^{n-1 / 2} \quad \text { for } \quad n \geq 2
\end{aligned}
$$

and

$$
q^{1}(\phi)=\tau_{10} \phi^{0}=\int_{0}^{t_{1}} K\left(t_{1}-s\right) d s \phi^{0}
$$

where

$$
\tau_{n j}= \begin{cases}\int_{t_{j}}^{t_{j+1}} K\left(t_{n}-s\right) d s & \text { if } \quad j \leq n-2, \\ \int_{t_{n}-1}^{t_{n}} K\left(t_{n}-s\right) \frac{t_{n-1 / 2}-s}{k_{n-1 / 2}} d s & \text { if } \quad j=n-1, \\ \int_{t_{n-1}}^{t_{n}} K\left(t_{n}-s\right) \frac{s-t_{n-3 / 2}}{k_{n-1 / 2}} d s & \text { if } \quad j=n .\end{cases}
$$

Our fully discretized scheme based on the Crank-Nicolson scheme is now defined by

$$
\begin{aligned}
\left(\bar{\partial}_{t} U^{n}, \chi\right)+A\left(U^{n-1 / 2}, \chi\right) & =q^{n-1 / 2}(B(U, \chi))+\left(f^{n-1 / 2}, \chi\right), \quad n \geq 2, \\
\left(\bar{\partial}_{t} U^{1}, \chi\right)+A\left(U^{1}, \chi\right) & =q^{1}(B(U, \chi))+\left(f^{1}, \chi\right),
\end{aligned}
$$

where

$$
q^{n-1 / 2}(B(U, \chi))=\frac{1}{2}\left\{q^{n}(B(U, \chi))+q^{n-1}(B(U, \chi))\right\} .
$$

The purpose of this paper is to show stability and to obtain error estimates for the scheme (1.8).

\section{Stability and convergence}

We show stability and obtain the error estimates for the fully discretized scheme (1.8). The following three lemmas from Kim and Choi [5] are required for our analysis.

LEMMA 1. If the grading exponent $r>1$, then we have the following estimates. There is a positive constant $C$ dependent on $T$ and $r$ such that 
(i) $\left|\tau_{n j}\right| \leq C(n-j)^{-\alpha} k_{j+1}^{1-\alpha}$,

(ii) $\sum_{n=2}^{M} \sum_{j=1}^{n}\left|\tau_{n j}-\tau_{n-1 j-1}\right| \leq C$.

LEMMA 2. For each $\epsilon>0$, there is a constant $C_{\epsilon}$ such that

$\left|\sum_{l=1}^{n} k_{l} \sum_{j=0}^{l-1}(n-j)^{-\alpha} k_{j+1}^{1-\alpha} f_{j} f_{l}\right| \leq \epsilon \sum_{l=1}^{n} k_{l} f_{l}^{2}+C_{\epsilon} \sum_{l=0}^{n-1}(n-l)^{-\alpha} k_{l+1}^{1-\alpha} \sum_{j=0}^{l} k_{j+1} f_{j}^{2}$.

Also we need the following discrete version of the Gronwall lemma.

LEMMA 3. Let $\left\{w_{n}\right\}$ be a sequence of nonnegative real numbers satisfying

$$
w_{n} \leq \beta_{n}+\sum_{j=0}^{n-1}(n-j)^{-\alpha} k_{j+1}^{1-\alpha} w_{j}
$$

where $\beta_{n} \geq 0$. Then there is a positive constant $C$ such that

$$
w_{n} \leq \beta_{n}+C \sum_{j=0}^{n-1}(n-j)^{-\alpha} k_{j+1}^{1-\alpha} \beta_{j}, \quad n \geq 0
$$

We recall that the bilinear form $A(\cdot, \cdot)$ is coercive and bounded if there are positive constants $c_{0}$ and $c_{1}$ satisfying

$$
c_{0}\|u\|_{1}^{2} \leq A(u, u) \leq c_{1}\|u\|_{1}^{2} \quad \forall u \in H^{2} \cap H_{0}^{1}
$$

The following theorem is our conditional stability result for the fully discretized scheme (1.8).

THEOREM 1. Suppose that $\bar{k}:=\max _{n \leq M} k_{n}$ is so small that

$$
\lambda:=\int_{0}^{\bar{k}}|K(s)| d s<\frac{c_{0}}{2\|B\|} \text { for all } n \geq 2
$$

Then scheme (1.8) is stable, that is, there is a positive constant $C_{T}:=C(T, r, \gamma)$ such that

$$
\left\|U^{n}\right\| \leq C_{T}\left(\left\|U^{0}\right\|+\sum_{j=1}^{n} k_{j}\left\|f^{j}\right\|\right) \quad \text { for } n \geq 1
$$


Proof. Letting $\tilde{U}^{n}=U^{n-1 / 2}$ for $n \geq 2, \tilde{U}^{0}=U^{0}$ and $\tilde{U}^{1}=U^{1}$, (i) of Lemma 1 implies that

$$
\begin{aligned}
& \left|q^{1}(B(U, \phi))\right| \leq C k_{1}^{1-\alpha}\left\|U^{0}\right\|_{1}\|\phi\|_{1} \\
& \left|q^{n}(B(U, \phi))\right| \leq C\left(\sum_{j=0}^{n-1}(n-j)^{-\alpha} k_{j+1}^{1-\alpha}\left\|\tilde{U}_{j}\right\|_{1}\|\phi\|_{1}\right)+\lambda\|B\|\left\|\tilde{U}^{n}\right\|_{1}\|\phi\|_{1}, \quad n \geq 2
\end{aligned}
$$

Taking $\phi=\tilde{U}^{n}$ for $n \geq 1$ in (1.8), we have

$$
\begin{aligned}
& \frac{1}{2}\left(\left\|U^{n}\right\|^{2}-\left\|U^{n-1}\right\|^{2}\right)+k_{n}\left(c_{0}-\lambda\|B\|\right)\left\|\tilde{U}^{n}\right\|_{1}^{2} \\
& \quad \leq C k_{n}\left\|\tilde{U}^{n}\right\|_{1}\left\{\sum_{j=0}^{n-1}(n-j)^{-\alpha} k_{j+1}^{1-\alpha}\left\|\tilde{U}^{j}\right\|_{1}\right\}+k_{n}\left\|f^{n-1 / 2}\right\|\left\|\tilde{U}^{n}\right\| \text { for } n \geq 2 .
\end{aligned}
$$

Summing (2.3) from $n=1$ to $N$ and applying Lemma 2 with a suitable $\epsilon$, we obtain

$$
\begin{aligned}
\left\|U^{N}\right\|^{2}+\sum_{n=1}^{N} k_{n}\left\|\tilde{U}^{n}\right\|_{1}^{2} \leq & \left\|U^{0}\right\|^{2}+C\left(k_{1}\left\|f^{1}\right\|\left\|U^{1}\right\|+\sum_{n=2}^{N} k_{n}\left\|f^{n-1 / 2}\right\|\left\|\tilde{U}^{n}\right\|\right) \\
& +C \sum_{n=1}^{N-1}(N-n)^{-\alpha} k_{n+1}^{1-\alpha} \sum_{j=0}^{n-1} k_{j+1}\left\|\tilde{U}^{j}\right\|_{1}^{2} .
\end{aligned}
$$

It follows from Lemma 3 that

$$
\left\|U^{N}\right\|^{2}+\sum_{n=1}^{N} k_{n}\left\|\tilde{U}^{n}\right\|_{1}^{2} \leq C_{T}\left\{\left\|U^{0}\right\|+k_{1}\left\|f^{1}\right\|+\sum_{n=2}^{N} k_{n}\left\|f^{n-1 / 2}\right\|\right\} \max _{n \leq N}\left\|U^{n}\right\| .
$$

Hence we have

$$
\left\|U^{N}\right\| \leq \max _{n \leq N}\left\|U^{n}\right\| \leq C_{T}\left(\left\|U^{0}\right\|+k_{1}\left\|f^{1}\right\|+\sum_{n=2}^{N} k_{n}\left\|f^{n-1 / 2}\right\|\right) \quad \text { for } 1 \leq N \leq M .
$$

Next we derive the error estimate for the fully discretized scheme (1.8). For the analysis, we introduce the "discrete Ritz-Volterra projection" $V_{h}$ defined for an appropriately smooth function $u$ by

$$
A\left(\left(V_{h} u-u\right)\left(t_{n}\right), \chi\right)=q^{n}\left(B\left(V_{h} u-u, \chi\right)\right), \quad \forall \chi \in S_{h} \text { for } n \geq 0 .
$$

We have the following two lemmas which state the error estimate for the discrete Ritz-Volterra projection (2.4). 
LEMMA 4. Assume that $\bar{k}:=\max _{n \leq M} k_{n}$ is so small that

$$
\lambda:=\int_{0}^{\bar{k}}|K(s)| d s<\frac{c_{0}}{\|B\|} \text { for all } n \geq 2
$$

and that $u(t) \in H^{2} \cap H_{0}^{1}$ and $u(t) \in C^{2}(\Omega)$. Then we have for $V_{h}$ that

$$
\begin{aligned}
\left\|\left(V_{h} u^{n}-u^{n}\right)\right\| & \leq C_{T} h^{2} \sup _{j \leq n}\left\|u^{j}\right\|_{2} \\
& \leq C_{T} h^{2}\left(\left\|u_{0}\right\|_{2}+\int_{0}^{t_{n}}\left\|u_{t}(s)\right\|_{2} d s\right) .
\end{aligned}
$$

PROOF. Let $\rho=V_{h} u-u$ and let $R_{h}$ be the Ritz projection defined by

$$
A\left(R_{h} u-u, \chi\right)=0, \quad \forall \chi \in S_{h} .
$$

It is a well-known estimate for $R_{h}$ that

$$
\left\|\left(R_{h} u-u\right)(t)\right\|+h\left\|\left(R_{h} u-u\right)(t)\right\|_{1} \leq C h^{2}\|u(t)\|_{2} .
$$

Letting $\theta^{n}=V_{h} u^{n}-R_{h} u^{n}$ under the definition of $V_{h}$, we have that with $c_{0}>0$,

$$
\begin{aligned}
c_{0}\left\|\theta^{n}\right\|_{1}^{2} & \leq A\left(\theta^{n}, \theta^{n}\right)=A\left(\rho^{n}, \theta^{n}\right)=q^{n}\left(B\left(\rho, \theta^{n}\right)\right) \\
& \leq C_{r}\left\|\theta^{n}\right\|_{1} \sum_{j=0}^{n-1}(n-j)^{-\alpha} k_{j+1}\left\|\rho^{j}\right\|_{1}+\lambda\|B\|\left\|\rho^{n}\right\|_{1}\left\|\theta^{n}\right\|_{1},
\end{aligned}
$$

or

$$
\left(c_{0}-\lambda\|B\|\right)\left\|\rho^{n}\right\|_{1} \leq C\left\|R_{h} u^{n}-u^{n}\right\|_{1}+C_{T}\left(\sum_{j=0}^{n-1}(n-j)^{-\alpha} k_{j+1}^{1-\alpha}\left\|\rho^{j}\right\|_{1}\right) .
$$

Thus Lemma 3 implies that

$$
\left\|\rho^{n}\right\|_{1} \leq C_{T} \sup _{j \leq n}\left\|R_{h} u^{j}-u^{j}\right\|_{1} \leq C_{T} h \sup _{j \leq n}\left\|u^{j}\right\|_{2} .
$$

In order to obtain the $L_{2}$-estimate for $\rho^{n}$, we use a duality argument defined by

$$
\left\|\rho^{n}\right\|=\sup _{\|\phi\|=1}\left(\rho^{n}, \phi\right)
$$

For each such $\phi$, we let $\Psi$ be the solution of

$$
\mathscr{A} \Psi=\emptyset \text { in } \Omega, \quad \Psi=0 \text { on } \partial \Omega .
$$


Then, for $\chi \in S_{h}$, we have from (2.2) that

$$
\begin{aligned}
\left(\rho^{n}, \phi\right)= & A\left(\rho^{n}, \Psi\right)=A\left(\rho^{n}, \Psi-\chi\right)+A\left(\rho^{n}, \chi\right) \\
= & A\left(\rho^{n}, \Psi-\chi\right)+q^{n}(B(\rho, \chi-\Psi))+q^{n}\left(\left(\rho, \mathscr{B}^{*} \Psi\right)\right) \\
\leq & C\left\|\rho^{n}\right\|_{1}\|\Psi-\chi\|_{1}+C \sup _{j \leq n}\left\|\rho^{j}\right\|_{1}\|\chi-\Psi\|_{1} \\
& +C_{T} \sum_{j=0}^{n-1}(n-j)^{-\alpha} k_{j+1}^{1-\alpha}\left\|\rho^{j}\right\|+\lambda\left\|\mathscr{B}^{*} \Psi\right\|\left\|\rho^{n}\right\| .
\end{aligned}
$$

Take $\chi=R_{h} \Psi$ and note that $c_{0}\left\|\mathscr{A}^{-1}\right\| \leq 1$ is an easy consequence of (2.1) and thus $1-\lambda\left\|\mathscr{B}^{*} \Psi\right\| \geq \frac{1}{2}$. Then we obtain that

$$
\left\|\rho^{n}\right\| \leq C h \sup _{j \leq n}\left\|\rho^{j}\right\|_{1}+C_{T} \sum_{j=0}^{n-1}(n-j)^{-\alpha} k_{j+1}^{1-\alpha}\left\|\rho^{j}\right\| .
$$

Thus Lemma 3 implies $\left\|\rho^{n}\right\| \leq C h \sup _{j \leq n}\left\|\rho^{j}\right\|_{1}$, which completes the proof.

LEMMA 5. Under the assumptions of Lemma 4, we have for $\rho=V_{h} u-u$,

$$
\sum_{j=1}^{n} k_{j}\left\|\bar{\partial}_{t} \rho^{j}\right\| \leq C h^{2}\left\{\left\|u_{0}\right\|_{2}+\int_{0}^{t_{n}}\left\|u_{t}(s)\right\|_{2} d s\right\} .
$$

PROOF. For the sake of convenience, we denote $\delta^{n}=k_{n} \bar{\partial}_{t} \rho^{n}, \delta^{0}=\rho^{0}, \omega_{n j}=$ $\tau_{n j}-\tau_{n-1 j-1}, W_{n}=\sum_{j=1}^{n}\left|\omega_{n j}\right|$ for $n \geq 2$, and $W_{1}=W_{0}=0$. Then we obtain directly from (2.4) that for all $\chi \in S_{h}$,

$$
\begin{aligned}
A\left(\delta^{n}, \chi\right)= & k_{n} \bar{\partial}_{t} q^{n}(B(\rho, \chi)) \\
= & q^{n}(B(\delta, \chi))+\sum_{j=1}^{n-2} \omega_{n j} B\left(\rho^{j-1 / 2}, \chi\right) \\
& \quad+\omega_{n n-1} B\left(\rho^{n-5 / 2}, \chi\right)+\omega_{n n} B\left(\rho^{n-3 / 2}, \chi\right) \\
\leq & C_{T} \sum_{j=0}^{n-1}(n-j)^{-\alpha} k_{j+j}^{1-\alpha}\left|B\left(\delta^{j}, \chi\right)\right|+\lambda\left|B\left(\delta^{n}, \chi\right)\right|+C \max _{j \leq n}\left\|\rho^{j}\right\|_{1}\|\chi\|_{1} W_{n} .
\end{aligned}
$$

Taking $\theta^{n}=\bar{\partial}_{t}\left(V_{h} u^{n}-R_{h} u^{n}\right)$, we have with $c_{0}>0$,

$$
\begin{aligned}
c_{0} k_{n}\left\|\theta^{n}\right\|_{1}^{2} & =A\left(\delta^{n}, \theta^{n}\right) \\
& \leq C_{r}\left\|\theta^{n}\right\|_{1}\left\{\sum_{j=0}^{n-1}(n-j)^{-\alpha} k_{j+1}^{1-\alpha}\left\|\delta^{j}\right\|_{1}+\max _{j \leq n}\left\|\rho^{j}\right\|_{1} W_{n}\right\}+\lambda\|B\|\left\|\delta^{n}\right\|_{1} .
\end{aligned}
$$


Hence we get

$$
\left\|\delta^{n}\right\|_{1} \leq C_{T} \sum_{j=0}^{n-1}(n-j)^{-\alpha} k_{j+1}^{1-\alpha}\left\|\delta^{j}\right\|_{1}+C \max _{j \leq n}\left\|\rho^{j}\right\|_{1} W_{n}+C k_{n}\left\|\bar{\partial}_{t}\left(R_{h} u^{n}-u^{n}\right)\right\|_{1} .
$$

It follows from Lemma 3 that

$$
\begin{aligned}
k_{n}\left\|\bar{\partial}_{t} \rho^{n}\right\|_{1} \leq & C_{T} \max _{j \leq n}\left\|\rho^{j}\right\|_{1}\left(\sum_{j=0}^{n-1}(n-j)^{-\alpha} k_{j+1}^{1-\alpha} W_{j}+W_{n}\right) \\
& +C_{T} h \sum_{j=0}^{n-1}(n-j)^{-\alpha} k_{j+1}^{1-\alpha} \int_{t_{j}}^{t_{j+1}}\left\|u_{s}(s)\right\|_{2} d s .
\end{aligned}
$$

We can easily verify that for $\beta_{j} \geq 0$,

$$
\sum_{n=1}^{N} \sum_{j=0}^{n-1}(n-j)^{-\alpha} k_{j+1}^{1-\alpha} \beta_{j}=\sum_{j=0}^{N-1} \beta_{j} \sum_{n=j+1}^{N}(n-j)^{-\alpha} k_{j+1}^{1-\alpha} \leq C \sum_{j=0}^{N-1} \beta_{j} .
$$

Summing (2.6) from $n=1$ to $N$ and applying the inequality (2.7) and (ii) of Lemma 1 , we have

$$
\sum_{n=1}^{N} k_{n}\left\|\bar{\partial}_{t} \rho^{n}\right\|_{1} \leq C h\left(\max _{n \leq N}\left\|u^{n}\right\|_{2}+\int_{0}^{t_{N}}\left\|u_{s}(s)\right\|_{2} d s\right) .
$$

With the same argument of Lemma 4, we can write with $\chi=R_{h} \Psi$,

$$
\begin{aligned}
k_{n}\left(\bar{\partial}_{t} \rho^{n}, \phi\right) & =k_{n} A\left(\bar{\partial}_{t} \rho^{n}, \Psi\right)=k_{n} A\left(\bar{\partial}_{t} \rho^{n}, \Psi-\chi\right)+k_{n} A\left(\bar{\partial}_{t} \rho^{n}, \chi\right) \\
& =k_{n} A\left(\bar{\partial}_{t} \rho^{n}, \Psi-\chi\right)+k_{n} \bar{\partial}_{t} q^{n}(B(\rho, \chi-\Psi))+k_{n} \bar{\partial}_{t} q^{n}\left(\left(\rho, \mathscr{B}^{*} \Psi\right)\right) \\
& \leq G_{n}+C_{T} \sum_{j=0}^{n-1}(n-j)^{-\alpha} k_{j+1}^{1-\alpha}\left\|\delta^{j}\right\|+\lambda\left\|\mathscr{B}^{*} \Psi\right\|\left\|\rho^{n}\right\|,
\end{aligned}
$$

where

$$
\begin{aligned}
G_{n}:= & C h\left(k_{n}\left\|\bar{\partial}_{t} \rho^{n}\right\|_{1}+\sum_{j=0}^{n-1}(n-j)^{-\alpha} k_{j+1}^{1-\alpha}\left\|\delta^{j}\right\|_{1}\right) \\
& +C_{T}\left(h \max _{j \leq n}\left\|\rho^{j}\right\|_{1}+\max _{j \leq n}\left\|\rho^{j}\right\|\right) W_{n} .
\end{aligned}
$$

Thus we have

$$
\begin{aligned}
k_{n}\left\|\bar{\partial}_{t} \rho^{n}\right\| \leq & C h\left(k_{n}\left\|\bar{\partial}_{t} \rho^{n}\right\|_{1}+\sum_{j=0}^{n-1}(n-j)^{-\alpha} k_{j+1}^{1-\alpha}\left\|\delta^{j}\right\|_{1}\right) \\
& +C h^{2} \sup _{j \leq n}\left\|u^{j}\right\|_{2} W_{n}+C T \sum_{j=0}^{n-1}(n-j)^{-\alpha} k_{j+1}^{1-\alpha}\left\|\delta^{j}\right\| .
\end{aligned}
$$


Applying Lemma 4 again, summing from $n=1$ to $N$ and using the inequality (2.7) and Lemma 1, we obtain that

$$
\begin{aligned}
\sum_{n=1}^{N}\left\|\bar{\partial}_{t} \rho^{n}\right\| & \leq C h \sum_{j=1}^{N} k_{n}\left\|\bar{\partial}_{t} \rho^{n}\right\|_{1}+\max _{n \leq N}\left\|u^{n}\right\|_{2} \sum_{n=1}^{N} W_{n} \\
& \leq C h^{2}\left(\left\|u^{0}\right\|_{2}+\int_{0}^{t_{N}}\left\|u_{s}(s)\right\|_{2} d s\right),
\end{aligned}
$$

which completes the proof.

We also need the following error estimate for our quadrature scheme (1.7).

LEMMA 6. Suppose that $f \in C^{2}(0, T], f^{\prime} \in C[0, T]$ and $\left|f^{\prime \prime}(t)\right| \leq C t^{-\alpha}$. If a grading exponent $r \geq 2 /(2-\alpha)$, then there is a constant $C_{T}$ depending on $f$ and $T$ such that

$$
\left|\sum_{n=1}^{M} k_{n}\left(\int_{0}^{t_{k}} K\left(t_{k}-s\right) f(s) d s-q^{n}(f)\right)\right| \leq C_{T}(f) \bar{k}^{2} .
$$

PROOF. Refer to Kim and Choi [5].

Finally, we obtain the second-order convergence result for the fully discretized scheme (1.8).

THEOREM 2. Let $u$ and $\left\{U^{n}\right\}$ be the solution of (1.1) and (1.8) respectively. We assume that for sufficiently smooth data $u_{0}$ and $f$, u satisfies $u \in C\left([0, T] ; H^{2} \cap H_{0}^{1}\right) \cap$ $C^{3}\left((0, T] ; L^{2}(\Omega)\right), u_{t} \in L_{1}\left(0, T ; H^{2} \cap H_{0}^{1}\right)$ and $u_{t t} \in L_{1}\left(0, T ; H^{2}\right) \cap C^{1}\left((0, T] ; H^{2}\right)$. Furthermore, we assume that $\left\|u_{t t}\right\|_{2} \leq R_{0} t^{-\alpha}$ for some $R_{0}>0$. If a grading exponent $r$ is greater than $2 /(2-\alpha)$, then there exists a constant $C_{T}$ independent of $h$ and $k$ such that

$$
\left\|u^{n}-U^{n}\right\| \leq C_{T}(u)\left(h^{2}+\bar{k}^{2}\right) .
$$

ProOF. Let $\tilde{u}=V_{h} u$ for all $t_{k} \geq 0$ and $e^{n}=U^{n}-V_{h} u^{n}+V_{h} u^{n}-u^{n}=\theta^{n}+\rho^{n}$. Comparing (1.8) with the variational form of (1.1) and introducing (2.4) we have the following identity for $n \geq 2$ :

$$
\left(\bar{\partial}_{t} \theta^{n}, \phi\right)+A\left(\theta^{n-1 / 2}, \phi\right)=q^{n-1 / 2}(B(\theta, \phi))+I_{1}^{n}+I_{2}^{n},
$$

where we denote $I_{1}^{n}$ and $I_{2}^{n}$ as follows:

$$
\begin{aligned}
& I_{1}^{n}=\left(u_{t}^{n-1 / 2}, \phi\right)-\left(\bar{\partial}_{t} V_{h} u^{n}, \phi\right)=\left(u_{t}^{n-1 / 2}-\bar{\partial}_{t} u^{n}, \phi\right)-\left(\bar{\partial}_{t} \rho^{n}, \phi\right), \\
& I_{2}^{n}=q^{n-1 / 2}(B(u, \phi))-\frac{1}{2}\left(J_{n}(B(u, \phi))+J_{n-1}(B(u, \phi))\right) .
\end{aligned}
$$


With the same argument as that used in Theorem 1 and taking $\phi=\tilde{\theta}^{n}$ in (2.8), we have

$$
\begin{aligned}
& \frac{1}{2}\left(\left\|\theta^{n}\right\|^{2}-\left\|\theta^{n-1}\right\|^{2}\right)+k_{n}\left\|\tilde{\theta}^{n}\right\|_{1}^{2} \\
& \quad \leq C k_{n} \sum_{j=0}^{n-1}(n-j)^{-\alpha} k_{j+1}\left\|\tilde{\theta}^{j}\right\|_{1}\left\|\tilde{\theta}^{n}\right\|_{1}+C k_{n}\left|I_{1}^{n}+I_{2}^{n}\right| .
\end{aligned}
$$

Summing (2.9) from $n=1$ to $N$ and applying Lemma 2, we immediately obtain

$$
\begin{aligned}
& \left\|\theta^{N}\right\|^{2}+\sum_{n=1}^{N} k_{n}\left\|\tilde{\theta}^{n}\right\|_{1}^{2} \\
& \leq\left\|\theta^{0}\right\|^{2}+C \sum_{n=1}^{N-1}(N-n)^{-\alpha} k_{n+1}^{1-\alpha} \sum_{j=0}^{n} k_{j+1}\left\|\tilde{\theta}^{j}\right\|_{1}^{2}+C \sum_{n=1}^{N} k_{n}\left(\left|I_{1}^{n}\right|+\left|I_{2}^{n}\right|\right) .
\end{aligned}
$$

We now turn to the estimates for $I_{1}$ and $I_{2}$. Since $u \in C^{2}\left(\left(0, t_{1}\right] ; L_{2}\right)$, the Taylor formula with the integral form of the remainder implies that

$$
k_{1}\left|\left(u_{t}^{1}-\bar{\partial}_{t} u^{1}, \tilde{\theta}^{1}\right)\right| \leq k_{1}^{2}\left\|\theta^{1}\right\| \int_{0}^{t_{1}}\left\|u_{t t}\right\| d s \leq C(u) k_{1}^{2-\alpha}\left\|\tilde{\theta}^{1}\right\| .
$$

If $u \in C^{3}\left((0, T] ; L_{2}\right)$, then still by the Taylor formula, we get

$$
\begin{aligned}
k_{n}\left|\left(u_{t}^{n-1 / 2}-\bar{\partial}_{t} u^{n}, \tilde{\theta}^{n}\right)\right| & \leq k_{n}^{2}\left\|\tilde{\theta}^{n}\right\| \int_{t_{n-1}}^{t_{n}}\left\|u_{t t}\right\| d s \\
& \leq C_{T}(u) k_{n}^{3} t_{k}^{-1-\alpha} \text { for } n \geq 2 .
\end{aligned}
$$

We can easily verify that

$$
k_{n} \leq r \frac{T}{M}\left(\frac{n}{M}\right)^{r-1} \text { for } n \leq M \quad \text { and } \quad \bar{k} \geq r\left(\frac{1}{2}\right)^{r-1} \frac{T}{M} .
$$

Denoting $r=2+p /(2-\alpha)$ for some $p>0$, we immediately have from (2.11)(2.13)

$$
\sum_{n=1}^{N} k_{n}\left|I_{1}^{n}\right| \leq \max _{n \leq N}\left\|\theta^{j}\right\|\left\{C_{T}(u)\left(\bar{k}^{2}+\bar{k}^{2} \sum_{n=2}^{N} \frac{1}{M}\left(\frac{n}{M}\right)^{-1+p}\right)+\sum_{n=1}^{N} k_{n}\left\|\bar{\partial}_{t} \rho^{n}\right\|\right\} .
$$

Also the estimate for $I_{2}^{n}$ is directly obtained by Lemma 6 :

$$
\sum_{n=1}^{N} k_{n}\left|I_{2}^{n}\right| \leq C_{T}(u) \bar{k}^{2} \max _{n \leq N}\left\|\theta^{n}\right\| .
$$

Thus, from Lemma 3 and Lemma 5, we obtain

$$
\left\|\theta^{n}\right\| \leq \max _{n \leq N}\left\|\theta^{n}\right\| \leq C h^{2}\left\{\left\|u_{0}\right\|_{2}+\int_{0}^{t_{n}}\left\|u_{t}\right\|_{2} d s\right\}+C_{T}(u) \bar{k}^{2} .
$$

Since the estimate for $\left\|\rho^{n}\right\|$ is given in Lemma 5, we complete the proof of the theorem. 


\section{Acknowledgement}

This work has been partially supported by Kon-Kuk University.

\section{References}

[1] H. Brunner, "Polynomial spline collocation methods for Volterra integro-differential equations with weakly singular kernels", IMA J. Numer Anal. 6 (1986) 221 239.

[2] C. Chen, V. Thomée and B. Wahlbin, "Finite element approximation of parabolic integrodifferential equation with a weakly singular kernel", Math. Comp. 58 (1992) 587-602.

[3] H. Kaneko and Y. Xu, "Gauss-type quadratures for weakly singular integrals and their application to Fredholm integral equations of the second kind", Math. Comp. 62 (1994) 725-738.

[4] C. H. Kim and U. J. Choi, "Spectral collocation methods for a partial integro-differential equation with a weakly singular kernel", J. Aust. Math. Soc. B 39 (1998) 408-430.

[5] C. H. Kim and U. J. Choi, "Time discretization with collocation methods for the parabolic partial integro-differential equation with weakly singular kernel", IMA J. Numer. Anal., submitted for publication.

[6] A. K. Pani, S. K. Chung and R. S. Anderssen, "On convergence of finite difference scheme for a parabolic generalized solutions of parabolic and hyperbolic integro-differential equations", Centre for Mathematics and its Application, The Australian National University, 1991, CMA Report CMA-MR3-91.

[7] A. K. Pani, V. Thomée and L. B. Wahlbin, "Numerical methods for hyperbolic and parabolic integro-differential equations", Journal of Integral Equations and Applications 4 (1992) 533-583.

[8] M. Renardy, W. J. Hrusa and J. A. Nohel, Mathematical problems in viscoelasticity, Pitman Monographs and Surveys in Pure and Applied Mathematics (Willey, New York, 1987).

[9] I. H. Sloan and V. Thomée, "Time discretization of an integro-differential equation of parabolic type", SIAM J. Numer. Anal. 23 (1986) 1052-1061.

[10] V. Thomée and N. Y. Zhang, "Error estimates for semidiscrete finite element methods for parabolic integro-differential equations", Math. Comp. 53 (1989) 121-139.

[11] E. G. Yanik and G. Fairweather, "Finite element methods for parabolic and hyperbolic partial integro-differential equation", Nonlinear Anal. 12 (1988) 785-809. 